\title{
Wireless sensor network calibration technique for low-altitude unmanned aerial vehicle localization in paddy field
}

\author{
Azhar Jaafar, Norashikin M. Thamrin, Noorolpadzilah Mohamed Zan \\ Faculty of Electrical Engineering, Universiti Teknologi MARA, UiTM Shah Alam, Selangor, Malaysia
}

\section{Article Info}

Article history:

Received Apr 6, 2020

Revised Jun 13, 2020

Accepted Jul 31, 2020

\section{Keywords:}

Distance measurement

RF signal

Signal strength

Unmanned aerial vehicle

Wireless sensor network

ABSTRACT

This paper presents the use of the received signal strength indicator (RSSI) from the RF signal to estimate the distance from a point where the signal is transmitted to the point where the signal is received. This can be a challenge as in the paddy field, the watery and dry conditions, as well as the height of the paddy plant can affect signal transmission during this estimation process. Two low-cost ground beacons, Beacon1 and Beacon2 (The coordinator), are used and placed in a known location with a fixed distance across the paddy field, which becomes the reference point during the distance estimation for the unmanned aerial vehicle (UAV). These signals are analyzed by using the non-right-angle trigonometry computation, to estimate the distance between the transmitter and the receiver. The estimated distance is compared with the measured value to determine the efficiency of this approach. The calibration trendlines of these beacons in the open, watery and dry paddy fields are discussed and presented. It is found that the dry paddy field gives less RSSI mean error and proved that humidity can contribute to the distance estimation error
\end{abstract}

This is an open access article under the CC BY-SA license.

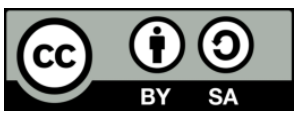

Corresponding Author:

Norashikin M. Thamrin, Faculty of Electrical Engineering, Universiti Teknologi MARA, 40450 Shah Alam, Selangor, Malaysia.

Email: norashikin@uitm.edu.my

\section{INTRODUCTION}

The breakthrough in wireless sensor network (WSN) has made the use of the RSSI signal to become the main focus in range measurement by the researchers [1] either for industry or domestic application [2, 3]. This technique integrates the wireless network with sensors and other embedded systems that can give valuable information [4] either for indoor [5-8] or outdoor environment [9, 10]. The WSN using the RSSI technique also increase the mobility and the coverage of the sensor's area [11]. The RSSI signal as a range measurement technique faces a huge challenge. The effect from the signal noise, signal refraction, reflection, multipath propagation and other interferences may have a great impact on the received RSSI signal [12]. Therefore, in real-time applications with the RSSI, the signal must have an accurate and reliable calibration technique to ensure that the received RSSI signal is a high-quality signal and stable [5].

A tracking strategy to calibrate the position of the XBee wireless adaptor has been proposed $[12,13]$. With the help of the trilateration algorithm and the least mean square (LMS) method, a grid for defining the possible locations of the XBee is built. Error correction is minimizing using the grid correction method [12, 14]. The limitation of this technique is when the localization error is bigger than the grid step size, the correction strategy becomes inefficient. To overcome this issue, the environmental factor is introduced as the environmental coefficient in the transmitter [15]. Based on this technique, an initial estimated object location value must be given and the distances between the object location and the transmitter are used together with the environmental corrected distance between the transmitters and the object in estimating its localization [6]. 
The disadvantage of this technique is that there must be at least 3 transmitters to transmit the RSSI values to the object receiver. Interesting work has been done by implementing a fingerprint-based location determination using the cascade artificial neural network (ANN) [16]. In this method, the dimension area covered in the experiment is quite small and more works need to be done and adjusted to be readily implemented in a larger area. Using the fingerprint-based system, the algorithm requires too much computation effort but resulting a great efficiency. This technique requires a database to collect and process the fingerprint data. In another method, combined the fingerprint-based system is combined with an improved trilateration method that is called as a unilateral technique [17]. Even though this technique works very well, undeniably this technique requires a lot of WSN in the area for the system to function.

The available systems as discussed previously are focusing on a very complicated system and use a lot of resources. On top of that, an excessive algorithm is needed to process the complex conditions. Therefore, in this paper, the RSSI based localization is introduced by using the minimum resources between the transmitter and the receiver. The RSSI signal on the wireless adaptor calibration is presented and evaluated and so that, the distance estimation by using this signal is obtained with a minimal error.

\section{RESEARCH METHOD}

In this research, a system based on the RF signal has been chosen. The system is controlled by using the Arduino Uno R3 microcontroller development platform. For the RF signal at the transmitter and receiver channels, the XBee wireless modules are used. The XBee modules are based on the IEEE 802.15.4-2003 standard that is designed to work on the wireless network for point to point communications and star communications for baud rates of $250 \mathrm{kbit} / \mathrm{s}$. The system is divided into 2 parts, namely, the former is set as the coordinator that is replicating the onboard UAV and the latter part is becoming the beacon. Each part is equipped with a set of Arduino Uno R3 microcontroller board with the XBee module.

The system architecture for the calibration technique is depicted as in Figure 1. Referring to this figure, the architecture of the system is developed by using two XBee Pro S1 modules, which one acts as the transmitter and the other one serves as the receiver. The transmit module is called Beacon1, and the other module, which calls the signal is called as Beacon2 or coordinator. The coordinator works as the main controller for the whole WSN architecture. The coordinator calls the beacon whenever it needs to read the RSSI signal. Both XBee modules in the coordinator and Beacon1 are controlled using the Arduino Uno R3 microcontroller.

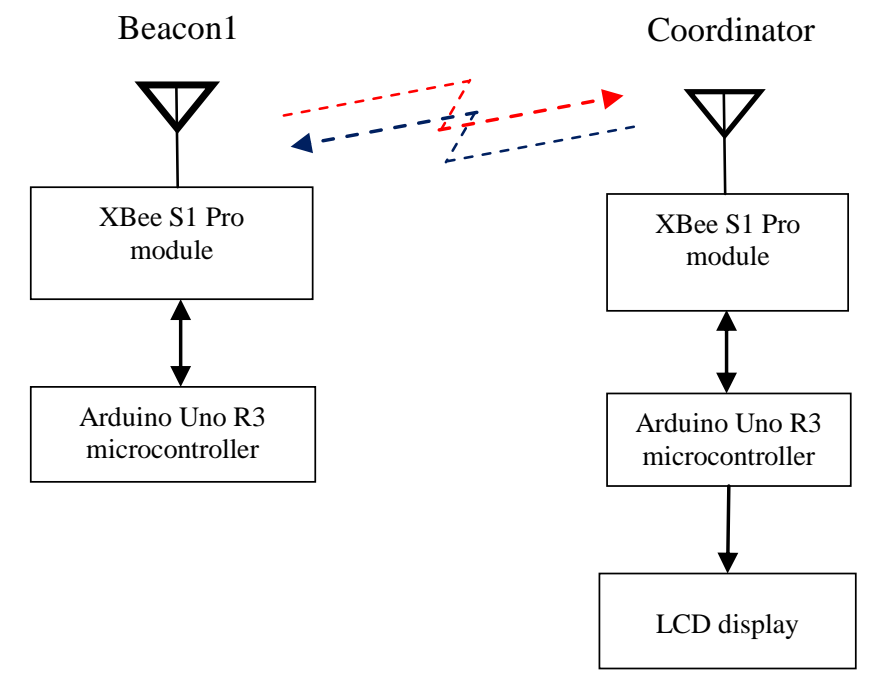

Figure 1. The architecture between the coordinator and Beacon1

Table 1 shows the configurations of the Arduino Uno R3 development board in this implementation. There are different setup for the coordinator and Beacon1. Basically, both of the controller boards are installed with the expansion Arduino board to easily interface with the Arduino development board with the XBee modules. As the whole system is not connected directly with any personal computer or laptop, therefore, a 16x2 array liquid crystal display (LCD) is used to show all the signals information on the related boards. To make possible for the wireless system configuration to be used wirelessly and portable,

Wireless sensor network calibration technique for low-altitude unmanned aerial... (Azhar Jaafar) 
both the coordinator and Beacon1 use rechargeable batteries as the main power source. Table 1 shows the specifications of the Arduino Uno R3 microcontroller.

Referring to this table, the Arduino Uno R3 uses 5 Volts as its main power supply and supports the voltage needed by the whole system. The small current usage by the microcontroller platform gives the advantage to the UAV as the power supply is very critical for the UAV implementation. The XBee Pro S1 provides RF communications within the range of approximately 100 meters. It has the ability of WSN, which can be set point to point communication or mesh network [18]. The performance of the XBee Pro S1 is shown in Table 2.

Table 1. The specifications of the Arduino Uno R3 microcontroller board

\begin{tabular}{ll}
\hline Microcontroller & ATmega328P \\
\hline Operating Voltage & $5 \mathrm{~V}$ \\
Input Voltage (recommended) & $7-12 \mathrm{~V}$ \\
Input Voltage (limits) & $6-20 \mathrm{~V}$ \\
Digital I/O Pins & 14 (of which 6 provide PWM output) \\
Analogue Input Pins & 6 \\
DC Current per I/O Pin & $40 \mathrm{~mA}$ \\
DC Current for 3.3V Pin & $50 \mathrm{~mA}$ \\
Flash Memory & $32 \mathrm{~KB}$ (ATmega328) of which $0.5 \mathrm{~KB}$ used by bootloader \\
SRAM & $2 \mathrm{~KB}$ (ATmega328) \\
EEPROM & $1 \mathrm{~KB}$ (ATmega328) \\
Clock Speed & $16 \mathrm{MHz}$ \\
\hline
\end{tabular}

Table 2. Performance specifications of XBee Pro S1

\begin{tabular}{ll}
\hline Specification & XBee PRO S1 \\
\hline Standard & IEEE 802.15 .4 \\
Frequency & $2.4 \mathrm{GHz}$ ISM \\
Indoor/urban range & Up to $300 \mathrm{ft} .(90 \mathrm{~m})$ \\
Outdoor RF line-of-sight range & Up to $1 \mathrm{mile}(1600 \mathrm{~m})$ \\
Transmit power output (software selectable) & $63 \mathrm{~mW}(18 \mathrm{dBm})$ \\
RF data rate & $250,000 \mathrm{~b} / \mathrm{s}$ \\
Serial interface data rate (software selectable) & $1200 \mathrm{bps}-250 \mathrm{~kb} / \mathrm{s}$ (non-standard baud rates also supported) \\
Receiver sensitivity (typical) & $100 \mathrm{dBm}(1 \%$ packet error rate) \\
Supply voltage & $2.8-3.4 \mathrm{~V}$ \\
Transmit current (typical) & $250 \mathrm{~mA}(@ 3.3 \mathrm{~V})$ (150 mA for international variant) RPSMA module only. \\
\hline
\end{tabular}

According to Table 2, the signal can be received up to 1600 meters in the open space. The XBee Pro S1 board requires 3.3 Volts of voltage supply and it also has good receiver sensitivity. It can be set up using 2 types of configuration, either by using the Application Transparent (AT) mode or by using the Application Programming Interface (API) mode [19, 20]. Using the AT mode, the XBee can do a simple network communication between point to point of two XBee modules directly. This feature is useful to read any wireless sensor value from a distance. In this work, the API mode is selected. This mode allows for more complex communications between 1 or more XBee modules [21]. The user can detect the ID of the transmitter together with the content of the transmitted data. To use XBee communication, firstly, it must be configured by using the XCTU software [22]. Table 3 shows the network configuration and setting for both XBee modules for the coordinator and Beacon1.

Table 3. XCTU network configuration and setting for XBee coordinator module and XBee Beacon1

\begin{tabular}{|c|c|c|c|}
\hline No. & Functions & XBee Coordinator Module & XBee Beacon1 \\
\hline 1. & CH Channel & $\mathrm{C}$ & $\mathrm{C}$ \\
\hline 2. & ID PAN ID & 33332 & 33332 \\
\hline & DH Destination Address High & 0 & 0 \\
\hline 3. & DL Destination Address Low & FFFF & 1 \\
\hline 4. & MY 16-bit Source Address & 0 & 0 \\
\hline 5. & AP API enable & API enabled w/PPP [2] & API enabled w/PPP [2] \\
\hline
\end{tabular}

The Arduino Uno R3 and the XBee Pro S1 are then put together in a plastic box for easy management and carry. Figure 2(a) shows the coordinator system component and (b) Beacon1 system component in their respective casing. 


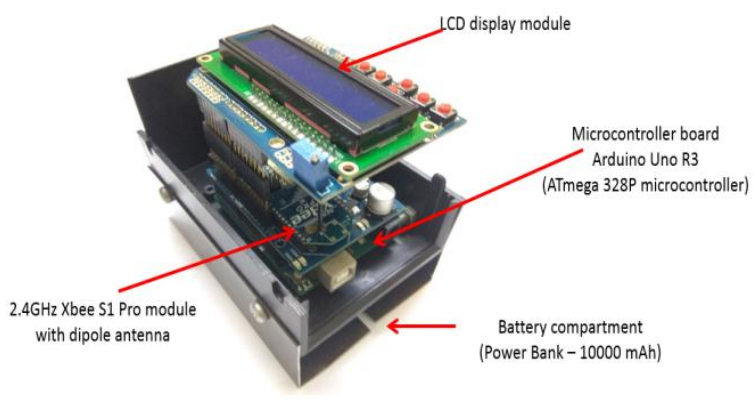

(a)

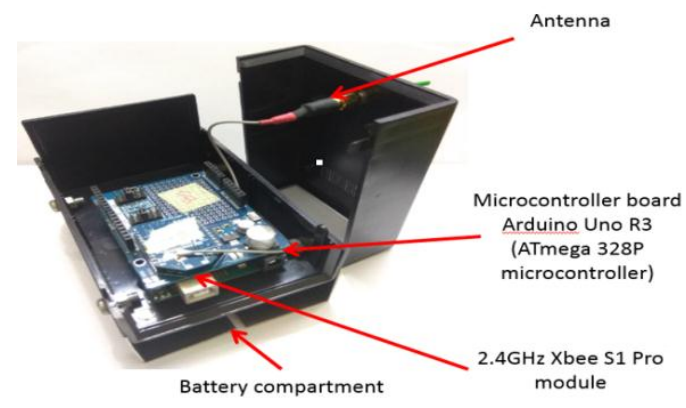

(b)

Figure 2. The complete hardware system for network calibration, (a) The coordinator hardware subsystem, (b) The Beacon1 hardware subsystem

\subsection{The signal calibration step}

The communication technique for system calibration is depicted in Figure 3. According to this figure, the whole calibration test is set up in the area that has a range of about 50 meters long. For a paddy field that has different field conditions, the tests were done in 3 different paddy field types, namely the dry paddy field, like a football field, a watery paddy field with the trees that were 1 meter above the ground and a dry paddy field with the matured paddy plant. In this setting, the coordinator XBee is the represents the UAV that will be moving freely on the paddy field. The XBee Beacon1 is the XBee module that has been placed on the ground at the start or the end of the paddy field. It acts as the on-ground beacon that stationed in that point of location and will transmit RF signal to the coordinator.

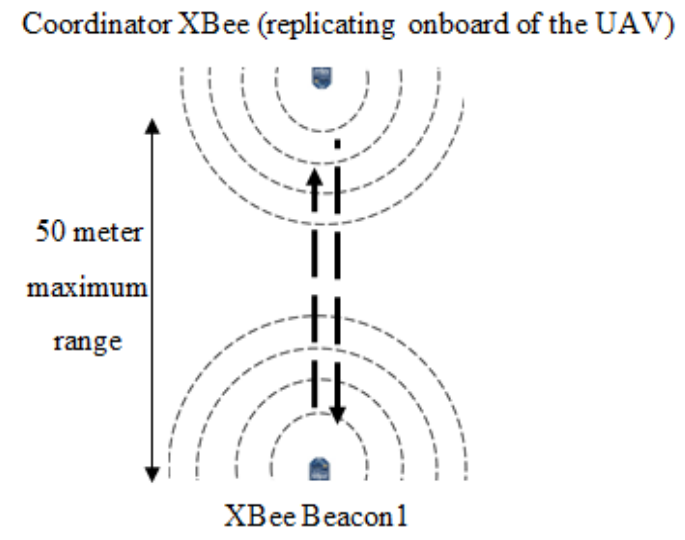

Figure 3. The conceptual framework of the calibration technique

The measurement is done in a straight-line measurement method. A measuring tape that has a length of more than 50 meters long is placed at the starting point in front of Beacon1. From this point, the measuring tape is pulled in a straight line forward from 0 meter to 50 meters. The coordinator and Beacon 1 are placed on top of a tripod stand that has a height of about 1.3 meters from the ground to represent the low-altitude UAV platform. Beacon1 is placed at the start of the measuring tape or 0-meter point. The coordinator is put in front of Beacon1 at the range of 1 meter. Then, the coordinator is moved along the measuring tape and stopped at every 1 meter of distance. RSSI value received by the coordinator is recorded at every stop. The flow of the sequence on how was the coordinator read the RSSI value from the Beacon1 and change it into range estimation in centimeter is shown in Figure 4.

The coordinator stores 10 RSSI values before taking the average value to reduce the detection error. This method is used to filter any spike that disturbs the RSSI efficiency which was read by the coordinator. Thus, for every ten RSSI values, one average RSSI value is produced. For calibration purposes, this signal then will be saved manually by the user and then the value will be calculated using regression analysis in MS Excel to create distance measurement. 


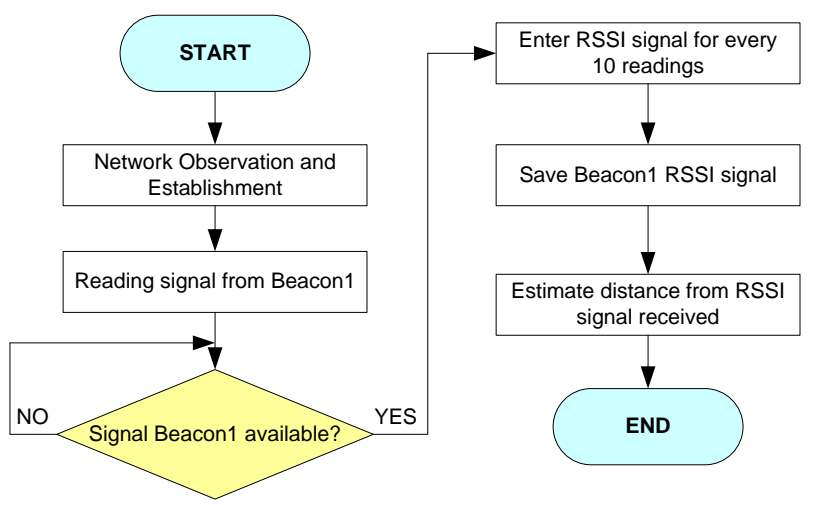

Figure 4. Flowchart of the coordinator reading and filtering the received RSSI value

\subsection{Regression analysis}

After the RSSI value is collected, the analysis of the data must be completed before starts formulate the equation that can be used for the calibration. Regression analysis is utilized to find a trend in the collected RSSI data. It can provide equation and prediction about the group of data [23, 24] and help to set accuracy to the data group. Figure 5 shows the process steps of the reference development technique for 1 beacon in one of the paddy field conditions. After building the graph according to the sequence in Figure 5, it is found that the graph is in the form of a logarithmic model. The logarithmic regression equations as given by the author in $[25,26]$ can be defined in a general form of (1).

$$
Y=A+(B) \ln x
$$

where,

$\mathrm{X}=$ Distance (meter)

$\mathrm{Y}=\mathrm{RSSI}$ value

From the graph, a trend line is plotted to normalize the available spiky form at different intervals of distance. The normalized value is in a modified form of the formula in (1). From this value, the mean of error is calculated to create a single reference value for the RSSI.

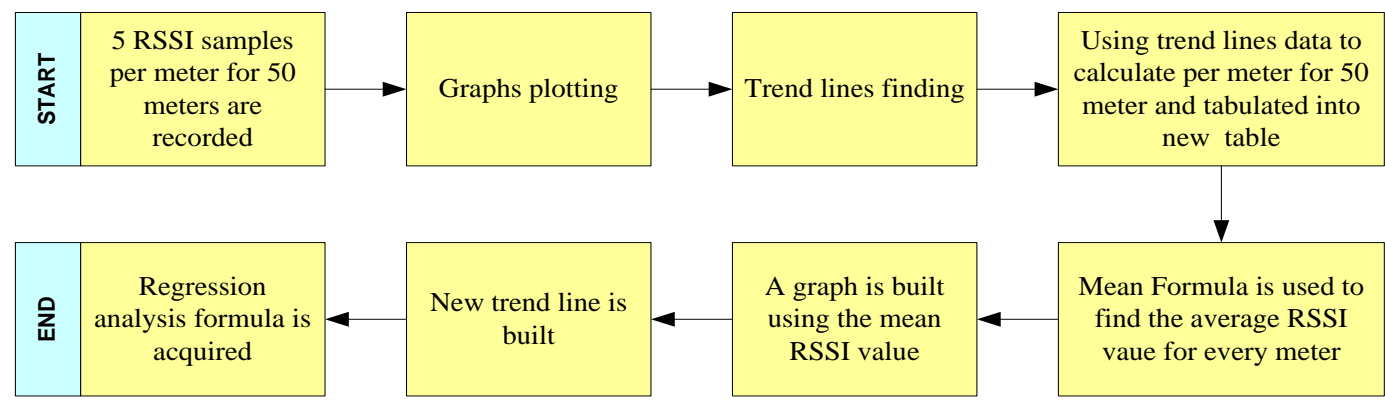

Figure 5. Process steps of the reference development technique for one beacon in one of the field conditions

\subsection{Distance range calculation}

The formula to calculate the range is found using the regression analysis as in (2). Using this formula, the distance between the coordinator and Beacon1 can be determined. This technique is used as the calibration value for a single beacon signal that is received by the coordinator.

$$
x_{1}(\text { Beacon } 1)=e^{\frac{-y_{1}-30.716}{6.161}}
$$

where,

$\mathrm{x} 1$ = Distance (meter) between coordinator and Beacon1

y1 = RSSI value from Beacon1 measured value 


\section{RESULTS AND DISCUSSION}

The RSSI signal calibration tests were executed to all 3 paddy field conditions that were mentioned earlier. A measuring tape is installed on the ground as the measurement guideline, which facilitates the comparison between the measured value and the estimated value obtained from the coordinator. The coordinator XBee is moved along the measuring tape line. For every 1 meter of range recorded using the measurement tape, the RSSI reading at the coordinator XBee that has been transmitted by Beacon1 is observed and recorded manually. Each one test will measure the signal strength of every 1 meter for the range of 50 meters. For the reliability purpose, every 1 meter of the movement of the coordinator, five readings have been taken. A total of 250 readings have been taken over the 50 meters of coordinator movement. Figures 6-8 show the RSSI signal that has been received from the test in an open field, dry and watery paddy field conditions. From these figures, five RSSI test signals and their trend line using regression analysis formula that have been extracted from the graph can be seen. From these trend lines, the mean values are calculated.

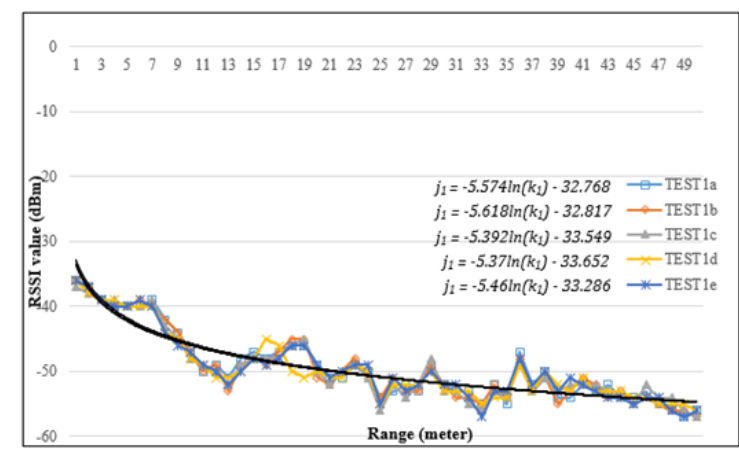

(a)

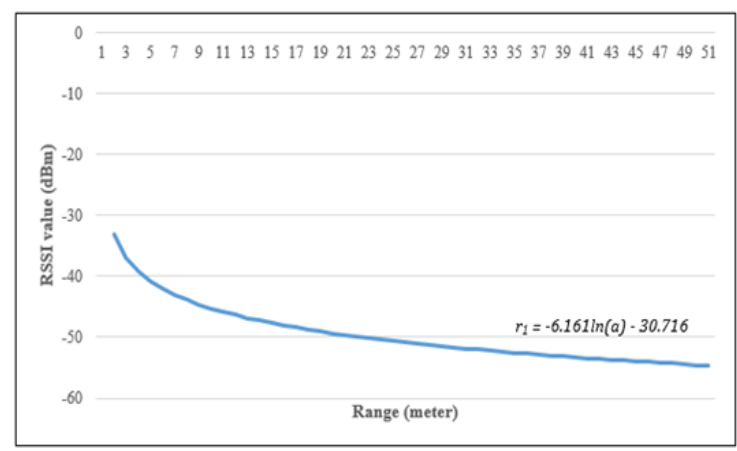

(b)

Figure 6. The result of RSSI signals tested at Beacon1 in open field condition, (a) The RSSI signal for five test values, (b) The RSSI mean value from five regression analysis calculations

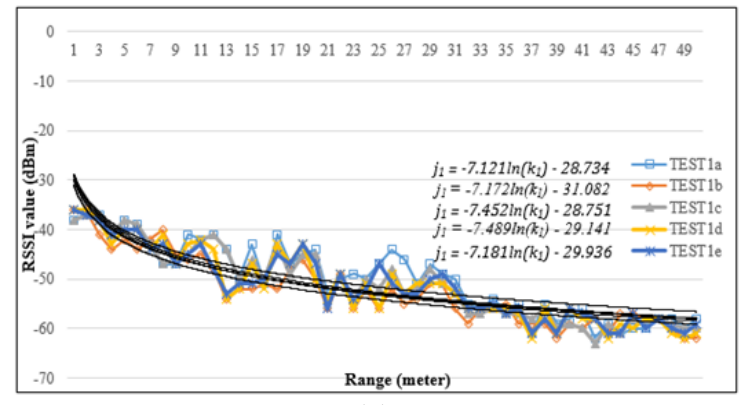

(a)

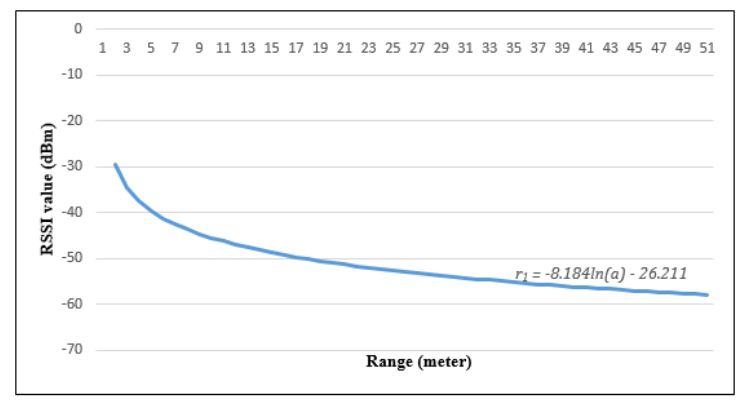

(b)

Figure 7. The result of RSSI signals tested at Beacon1 in dry paddy field condition, (a) The RSSI signal for five test values, (b) The RSSI mean value from five regression analysis calculations

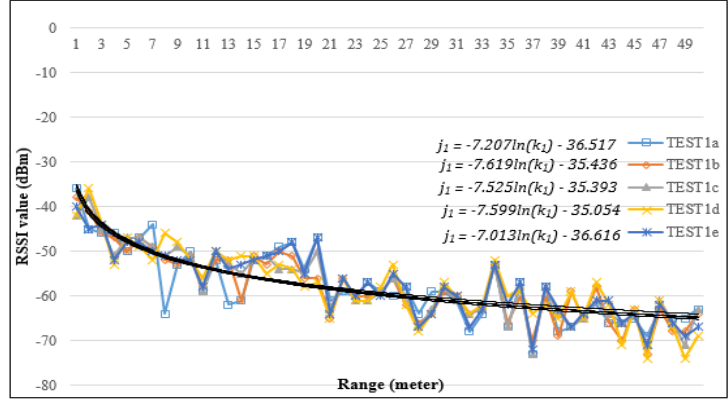

(a)

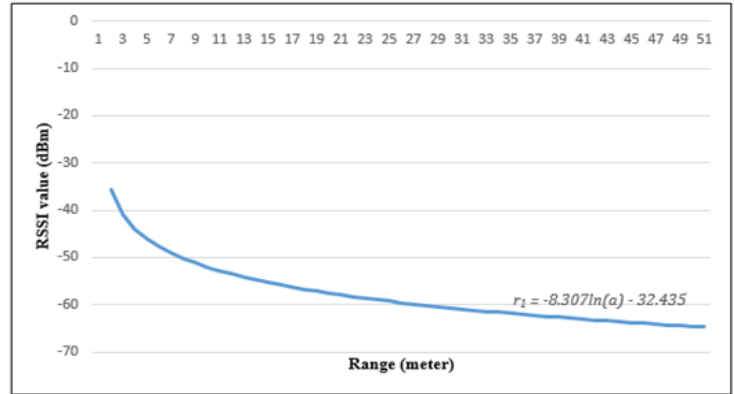

(b)

Figure 8. The result of RSSI signals tested at Beacon1 in watery paddy field condition, (a) The RSSI signal for five test values, (b) The RSSI mean value from five regression analysis calculations

Wireless sensor network calibration technique for low-altitude unmanned aerial... (Azhar Jaafar) 
Table 4 shows the reference value that has been used in calibration for the different types of fields. The field $\mathrm{r} 1$ represents the value of the RSSI signal and the calculated distance between the coordinator and Beacon1 in a meter. This formula is used in the Arduino Uno R3 algorithm to calibrate the distance between the coordinator and Beacon1. From these graphs, it shows that the distance estimation in the dry field has less error compared to the watery field by approximately 20 percent. This result is supported by the fact that humidity, which available in the watery field has disturbed the accuracy.

Table 4. The reference value for different open field type of paddy field

\begin{tabular}{lll}
\hline No. & Field condition & Coordinator formula \\
\hline 1 & Open field & $a=\exp \left(\frac{-r_{1}-30.716}{6.161}\right)$ \\
2 & Dry paddy field & $a=\exp \left(\frac{-r_{1}-26.211}{8.184}\right)$ \\
3 & Wet paddy field & $a=\exp \left(\frac{-r_{1}-32.435}{8.307}\right)$ \\
\hline
\end{tabular}

\section{CONCLUSION}

RSSI has been used as the medium of range measurement in this research. This calibration technique is targeted to be used as a measurement technique for measuring distance using the RSSI signal received by the XBee modules. The target of this research is to be deployed on a UAV platform that moves in a paddy planting field. In the end, the UAV can use this technique basically as their localization. Although a direct RSSI signal received can be used as range estimation, the effect of the environment on the received signals can confuse with the received signals. Using this calibration to the received signals, a more accurate and reliable range measurement can be obtained from the RSSI signals. Using a low-cost and lightweight component such as the Arduino Uno R3 and the XBee module will give the positive impact on the result. This technique is to help for more advance development base on the RSSI signal from the XBee in the future.

\section{ACKNOWLEDGEMENTS}

We thank Faculty of Electrical Engineering, Universiti Teknologi MARA and Politeknik Ungku Omar, for their support in terms of financial, facilities and knowledge.

\section{REFERENCES}

[1] H. H. Qasim, A. E. Hamza, H. H. Ibrahim, H. A. Saeed, and M. I. Hamzah, "Design and implementation home security system and monitoring by using wireless sensor networks WSN/internet of things IOT," International Journal of Electrical and Computer Engineering, vol. 10, no. 3, pp. 2617-2624, 2019.

[2] Jayasri B. S. and G. Raghavendra Rao, "Analytical modelling of power efficient reliable operation of data fusion in wireless sensor network," International Journal of Electrical and Computer Engineering, vol 8, no 6, pp. 4637-4645, 2018.

[3] F. Karray, M. Triki, M. W. Jamal, M. Abid, and A. M. Obeid, "WiRoTip: an IoT-based wireless sensor network for water pipeline monitoring," International Journal of Electrical and Computer Engineering, vol 8, no 5, pp.3250-3258, 2018.

[4] B. Guan and X. Li, "An RSSI-based wireless sensor network localization algorithm with error checking and correction," International Journal of Online and Biomedical Engineering, vol. 13, no. 12, pp. 52-66, 2017.

[5] W. Xue, W. Qiu, X. Hua, and K. Yu, "Improved Wi-Fi RSSI measurement for indoor localization," IEEE Sensors Journal, vol. 17, no. 7, pp. 2224-2230, 2017.

[6] S. Shue, L. E. Johnson, and J. M. Conrad, "Utilization of XBee ZigBee modules and MATLAB for RSSI localization applications," SoutheastCon 2017, pp. 1-6, 2017.

[7] S. Sadowski and P. Spachos, "RSSI-based indoor localization with the internet of things," IEEE Access, vol. 6, pp. 30149-30161, 2018

[8] L. Zhang, Z. Yang, S. Zhang, and H. Yang, "Three-dimensional localization algorithm of WSN nodes based on RSSI-TOA and single mobile anchor node," Journal of Electrical and Computer Engineering, pp. 1-8, 2019.

[9] K. Lam, C. Cheung, and W. Lee, "New RSSI-based LoRa localization algorithms for very noisy outdoor environment," 2018 IEEE 42nd Annual Comp. Soft. and Applica. Conference (COMPSAC), pp. 794-799, 2018.

[10] S. A. Arif, M. H. Niaz, N. Shabbir, M. H. Zafar, S. R. Hassan, and A. ur Rehman, "RSSI based trilatertion for outdoor localization in zigbee based wireless sensor networks (WSNs)," 2018 10th International Conference on Computational Intelligence and Communication Networks (CICN), pp. 1-5, 2018.

[11] B. Zhou, Q. Chen, and P. Xiao, "The error propagation analysis of the received signal strength-based simultaneous localization and tracking in wireless sensor networks," IEEE Transactions on Information Theory, vol. 63, no. 6, pp. 3983-4007, June 2017.

[12] J. Du, J-F. Diouris, and Y. Wang, "A RSSI-based parameter tracking strategy for constrained position localization," EURASIP Journal on Advances in Signal Processing, vol. 2017, no. 1, pp. 1-10, 2017. 
[13] M. Uradzinski, H. Guo, X. Liu, and M. Yu, "Advanced indoor positioning using zigbee wireless technology," Wireless Personal Communications, vol. 97, no. 4, pp. 6509-6518, 2017.

[14] A. Booranawong, J. Sopajarn, T. Sittiruk, and N. Jindapetch, "Reduction of RSSI variations for indoor position estimation in wireless sensor networks," Engineering and Applied Science Research, vol. 45, no. 3, pp. 212-220, 2018.

[15] Y. Liu, N. Li, D. Wang, T. Guan, W. Wang, J. Li, and Na Li, "Optimization algorithm of RSSI transmission model for distance error correction," Advances in Intelligent Information Hiding and Multimedia Signal Processing, Springer, pp. 27-36, 2020.

[16] E. Erdem, T. Tuncer, and R. Doğan, "Localization of a mobile device with sensor using a cascade artificial neural network-based fingerprint algorithm," International Journal of Computational Intelligence Systems, vol 12, no 1, pp. 238-249, 2018.

[17] V. Kaundal, P. Sharma, M. Prateek, and V. Chowdary, "Design, development and deployment of a RSSI based wireless network for post disaster management," International Journal Engineering \& Technology, vol. 7, no. 2, pp. 6-11, 2018.

[18] T. Sumalan, E. Lupu, R. Arsinte, and E. Onaca, "Multipoint wireless network for complex patient monitoring based on embedded processors," International Conference on Advancements of Medicine and Health Care through Technology, pp.123-126, 2016.

[19] J. Cheon, H. Hwang, D. Kim, and Y. Jung, "IEEE 802.15. 4 ZigBee-based time-of-arrival estimation for wireless sensor networks," Sensors, vol. 16, no. 2, p. 203, 2016.

[20] F. Tsvetanov, I. Georgieva, and M. Pandurski, "Topology influence on the radio communication range of sensor networks," Technium: Romanian Journal of Applied Sciences and Technology, vol. 2, no. 1, pp. 17-24, 2020.

[21] M. Postigo-Málaga, L. P. Figueroa, and J. Chilo, "Low cost outdoors WSN parking system for metropolitan areas based on RSS," 2019 10th IEEE International Conference on Intelligent Data Acquisition and Advanced Computing Systems: Technology and Applications (IDAACS), pp. 50-53, 2019.

[22] S. Soijoyo and A. Ashari, "Analysis of zigbee data transmission on wireless sensor network topology," International Journal of Advanced Computer Science and Applications, vol. 8, no. 9, pp. 145-151, 2017.

[23] Jamal I. Daoud, "Multicollinearity and regression analysis," Journal of Physics: Conference Series, vol. 949, no. 1 , p. $012009,2017$.

[24] A. F. Hayes and A. K. Monyota, "A tutorial on testing, visualizing, and probing an interaction involving a multicategorical variable in linear regression analysis," Communication Methods and Measures, vol. 11, no. 1, pp. 1-30, 2017.

[25] T. J. Cleophas and A. H. Zwinderman, "Logarithmic transformations, a great help to statistical analyses," Clinical Data Analysis on a Pocket Calculator, Springer, pp. 243-247, 2016.

[26] J. W. Yoon, H. Park, N. S. Kim, and T. Park, "Cost-effective, asynchronous inter-sensor distance estimation using trigonometry," Electronics Letters, vol. 52, no. 12, pp. 1080-1082, 2016. 